\title{
Cancer Epigenetics: It is Time to Move Forward to Therapy
}

\section{Taichun Qin*}

\section{Department of Cellular \& Molecular Oncology, University of Texas MD Anderson Cancer Center, Houston, TX 77030, USA}

Epigenetics is defined as the study of heritable changes in gene expression that do not involve changes in the DNA sequence. Epigenetic regulation of gene expression over the past few years has been recognized as an important role in the development of cancer. Two molecular mechanisms are known for epigenetic gene regulation: DNA methylation and histone modification [1]. DNA methylation is the addition of a methyl group to the cytosine $(\mathrm{C})$ in a cytosineguanine $(\mathrm{CpG})$ pair. "CpG islands" are the clusters of CpG pair in small stretches of DNA. These regions often regulate transcription of DNA into RNA. Almost half the genes in our genome have such CpG-rich promoter regions [2]. As with genetic aberration, aberrant promoter hypermethylation is an alternative mechanism to inactivate tumor suppressor genes. It can have an effect similar to coding-region mutation, leading to a loss of gene function.

Chemical modifications of the N-terminal domains of all core histones, such as acetylation, methylation, and phosphorylation at certain residues have been functionally linked to transcription and act as a 'histone code'. Transcription states can be predicted simply by deciphering this code [3,4]. Acetylation of lysine residues on the $\mathrm{N}$-terminal tails of the histones neutralizes the positive charge of the histone tail and decreases its affinity to negatively charged DNA, leading to the conversion of euchromatin from a compact to a more open structure. As a consequence, histone acetylation facilitates the binding of transcription factors to the promoter region to activate gene transcription [5], whereas deacetylation results in gene repression. These changes in acetylation are mediated by histone acetyltransferases [6] and histone deacetylases [7,8]. Growing evidence suggests a relationship between alterations in chromatin structure and the development of cancer. Histone methyltransferases (HMTs) are enzymes that catalyze the addition of methyl marks to histone proteins, as a result, it changes histone architecture and modulate gene expression. Aberrant expression of HMTs is associated with tumorigenesis and progression. The discovery of the first histone demethylase (HDM) that shows the reversibility of histone methylation also has an implication in cancer [9]. Lysine methyltransferases and demethylases are considered as transcriptional co-regulators that dynamically regulate the addition of histone methylation and removal of methylation. EZH2 is an important histone lysine methyltransferase that works cooperatively as part of a multi-subunit complex known as PRC2. EZH2 is responsible for methylating a key residue (H3K27) on histone tails.

Therefore, high expectations in the treatment are related to the use of so-called "epigenetic drugs" that reverse the aberrant epigenetic changes through modulating activity of enzymes. 5-aza-2'deoxycytidien and 5-azacytidine have been approved by the US food and drug administration for the treatment of myedysplastic syndrome and and some clinical trials of combining the demethylating agents with other anti-cancer drugs for the treatment of different types of lukemias and solid tumors are ongoing. Members of the superfamily of histone deacetylases (HDACs) are attractive targets for therapeutic intervention. HDAC inhibitors were therapeutically effective on hematological malignancies. For example, vorinostat and romidepsin have recently been approved for the treatment of relapsed or refractory T-cell lymphoma in the USA and Japan. The combination of DNA demethylaiton and HDACs are more efficient on activation of tumor suppressor genes, resulting in a better clinical outcome of therapy. EZH2 is over-expressed in many solid tumors and hematologic malignancies and typically associates with a poor prognosis as well as in tumor initiation and progression, migration and angiogenesis. GSK 126 that is highly selective for EZH2 that shows a promising perspective in the treatment of lymphoma with EZH2 mutations [10].

With the discovery of novel histone modifying enzymes that are involved in different types of cancer, it provides potential targets for drug development. Some pharmaceutical companies are working on modulating those enzymes to provide a potential better way for cancer treatment. We expect these novel approaches will benefit more cancer patients in the near future.

\section{References}

1. Jaenisch R, Bird A (2003) Epigenetic regulation of gene expression: how the genome integrates intrinsic and environmental signals. Nat Genet 33: 245-254

2. Robertson KD, Wolffe AP (2000) DNA methylation in health and disease. Nat Rev Genet 1: 11-19.

3. Kondo Y, Issa JP (2004) Epigenetic changes in colorectal cancer. Cancer Metastasis Rev 23: 29-39.

4. Jenuwein T, Allis CD (2001) Translating the histone code. Science 293: 1074 1080

5. Wolffe AP (1996) Histone deacetylase: a regulator of transcription. Science 272: $371-372$.

6. Roth SY, Denu JM, Allis CD (2001) Histone acetyltransferases. Annu Rev Biochem 70: 81-120.

7. Marks PA, Miller T, Richon VM (2003) Histone deacetylases. Curr Opin Pharmacol 3: 344-351

8. Yoshida M, Kijima M, Akita M, Beppu T (1990) Potent and specific inhibition of mammalian histone deacetylase both in vivo and in vitro by trichostatin A. J Biol Chem 265: 17174-17179.

9. Dawson MA, Kouzarides T (2012) Cancer Epigenetics: From Mechanism to Therapy. Cell 150: 12-27.

10. Michael TM, Heidi MO, Gopinath G, Susan K, Christine T, et al. (2012) $\mathrm{EZH} 2$ inhibition as a therapeutic strategy for lymphoma with EZH2-activating mutations. Nature 492: 108-112.

*Corresponding author: Taichun Qin, Department of Cellular \& Molecular Oncology, University of Texas MD Anderson Cancer Center, Houston, TX 77030, USA, Tel: 713-745-3931; Fax: 737-451-683; E-mail: qincharles@yahoo.com

Received November 30, 2012; Accepted December 02, 2012; Published December 08, 2012

Citation: Qin T (2012) Cancer Epigenetics: It is Time to Move Forward to Therapy J Bioanal Biomed S5: e001. doi:10.4172/1948-593X.S5-e001

Copyright: (c) 2012 Qin T. This is an open-access article distributed under the terms of the Creative Commons Attribution License, which permits unrestricted use, distribution, and reproduction in any medium, provided the original author and source are credited. 